\title{
Charge Carrier Heating Effect in Porous Silicon Structures Investigated by Microwaves
}

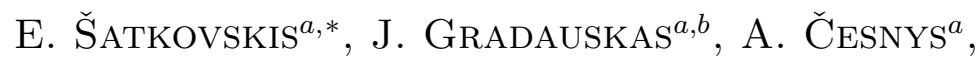 \\ J. StupAKOVA ${ }^{a}$ AND A. SuŽIEDE்LIS ${ }^{a}$ \\ ${ }^{a}$ Vilnius Gediminas Technical University \\ Saulètekio 11, LT-10223 Vilnius, Lithuania \\ ${ }^{b}$ Semiconductor Physics Institute
}

A. Goštauto 11, LT-01108, Vilnius, Lithuania

\begin{abstract}
Diode-like samples, containing porous silicon structures, were investigated by microwave radiation pulses. The resistance of the samples and electromotive force arising over the samples placed in a section of waveguide was measured. Reduction of resistance of the samples was observed with increase in microwave power. More complicated shape of the electromotive force dependence on pulse power was found. It is shown that both effects could be explained by models based on a concept of carrier heating by microwave radiation.
\end{abstract}

PACS numbers: 61.43.Gt, 78.70.Gq, 72.30.+q

\section{Introduction}

Because of the progress in semiconductor nanotechnology and miniaturization of semiconductor devices, the amplitudes of electric fields inevitably increase inside devices. As a result, charge carrier heating effects become more important. The heating is often investigated by microwave radiation (MW) [1]. This also applies to porous silicon (por-Si), one of the modern nanomaterials, which is intensively investigated at present (see, for example Ref. [2]). Till now, the action of MW radiation on por-Si remains almost unexplored. Basically there appeared papers where the application of por-Si in a role of substrates in microwave and opto-electronic interconnectors, due to low dielectric constant and high resistance of high porosity por-Si, is discussed $[1,3,4]$. Recently it has been shown that por-Si structures can be used as sensors of MW radiation not demanding any

*corresponding author; e-mail: eusat@fm.vgtu.lt 
external bias [5]. In the present paper, the effects related with hot carriers, electrical conductivity and electromotive force (emf) in por-Si structures are studied experimentally using high power MW radiation pulses.

\section{Experimental}

Bipolar (diode-like) samples containing layers of por-Si were fabricated by usual technology of electrochemical etching from plates the $0.4 \Omega \mathrm{cm}$ resistivity $p$-type silicon in the fluoric acid ethanol (in the ratio 1:1) electrolyte, and subsequent vacuum technologies of manufacturing of nonrectifying contacts $[1,6]$. Boron diffusion was performed on both surfaces of the initial plate before contact evaporation. Contacts of aluminium of $100 \mu \mathrm{m}$ in diameter were evaporated on the top of the plate through mask. Samples of size $0.5 \times 0.6 \mathrm{~mm}^{2}$ were cut from the plates. The current-voltage characteristics of the samples were linear at low voltages. The samples were placed in a section of the rectangular waveguide. Electric conductivity and emf were investigated under the action of MW radiation pulses of $10 \mathrm{GHz}$ frequency and $2 \mu$ s duration.

\section{Results and discussion}

With the increase in MW radiation pulse power $P$, the conductivity of the samples containing por-Si structures was found to grow (Fig. 1). The dependence of the resistance reduction $\Delta R=R_{\mathrm{MW}}-R_{0}$ on power is almost linear in the power range studied. Usually MW radiation causes heating of charge carriers in semiconductors and affects through their scattering processes [1]. The average mobility of the charge carriers decreases in strong MW fields as a result. Thus, we should

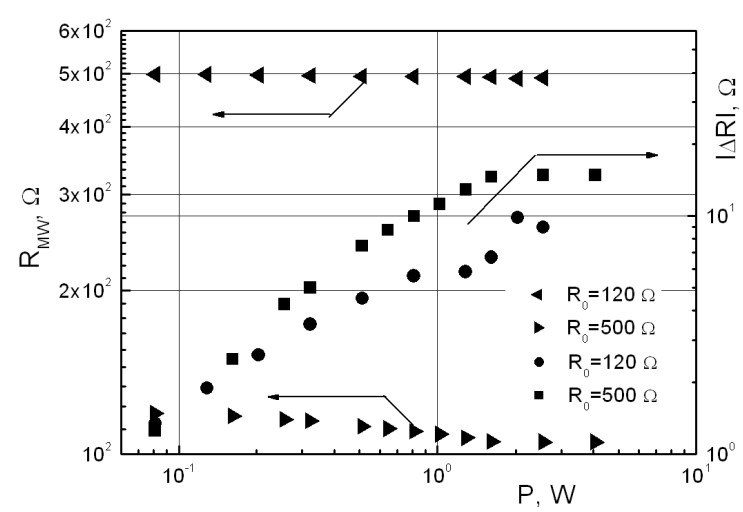

Fig. 1. Resistance $R$ and reduction of resistance $\Delta R$ of por-Si structures under the action of the MW radiation pulses. $R_{0}$ denotes the initial resistance of the sample.

expect the increases in the sample resistance if we follow this model. However, we observe experimentally an opposite picture: MW radiation stimulates conductivity in por-Si samples. The letter feature can be harmonized with activation model 
of electric conductivity in por-Si, suggested by Koch et al. [7]. The conductivity of por-Si according to activation model is

$$
\sigma(T) \sim \exp \left(-E_{0} / k T\right)
$$

where $E_{0}$ is some activation energy, $k$ is the Boltzmann constant, and $T$ is the ambient temperature. We believe that the MW radiation can stimulate the activation conductivity of por-Si through the enhancement of the hole temperature $T_{\mathrm{h}}$. After expanding the exponent (1) in series we get the following expression for $\Delta R$ :

$$
\Delta R \approx A^{\prime}\left[\left(1-E_{0}^{2} / 2 k T_{0} T_{\mathrm{h}}\right) \Delta T\right],
$$

where $a^{\prime}$ is some constant multiplier, $T_{0}$ is the lattice temperature, $\Delta T=T_{\mathrm{h}}-T_{0}$, $E_{0}=0.45 \mathrm{eV}[7]$. The sign of $\Delta R$ depends on the sign of the expression in parentheses. At $T_{0}=300 \mathrm{~K}$ we get $E_{0}^{2} / 2 k T_{0}=160$. Therefore, the expression in the parentheses is negative till $T_{\mathrm{h}} 5 \times 10^{4} \mathrm{~K}$. We not wave aside, however, the possibility of lowering potential barriers in por-Si by MW electric field.

The concept of carrier heating is suitable also to explain the origin of emf arising in the same por-Si structure exposed to MW radiation. Unlike the resistance, more complex shape of the emf dependence on pulse power $\mathrm{P}$, was found, Fig. 2. Initially, at low powers, emf signal is negative. If $P$ increases, the emf saturates, then drops to zero and turns positive.

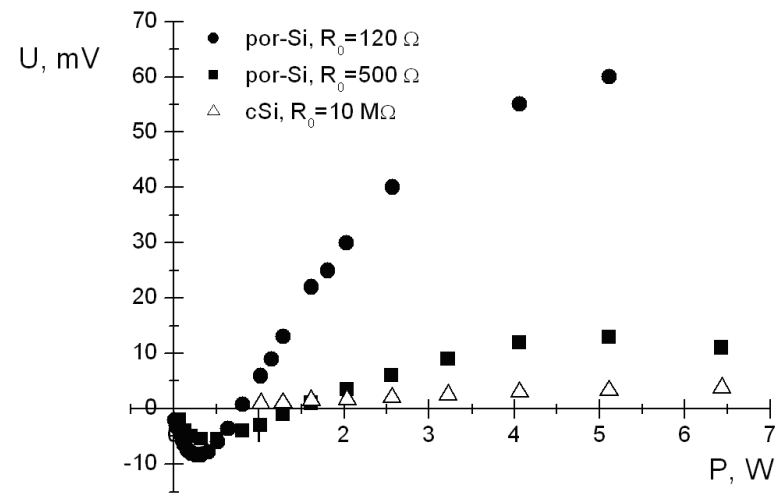

Fig. 2. Electromotive force of por-Si structures under action of MW radiation pulses.

In the last case the emf is similar to that observed in crystal Si (cSi, Fig. 3), which really occurs due to charge carrier heating effect [5]. The rise of negative emf can be explained by electric scheme of our samples, as equivalent to the scheme of two diodes, connected in opposite direction to each other. This assumption is explaned by energy band scheme across the sample presented in Fig. 3. It involves aluminium contacts on the both sides of the sample, highly doped low porosity por-Si layer $p^{+}$PSiL1, the basic high porosity por-Si layer PSiL2, part of initial crystalline plate $\mathrm{p}-\mathrm{cSi}$, and doped $p^{+} \mathrm{cSi}$ region. Two nearly similar potential 


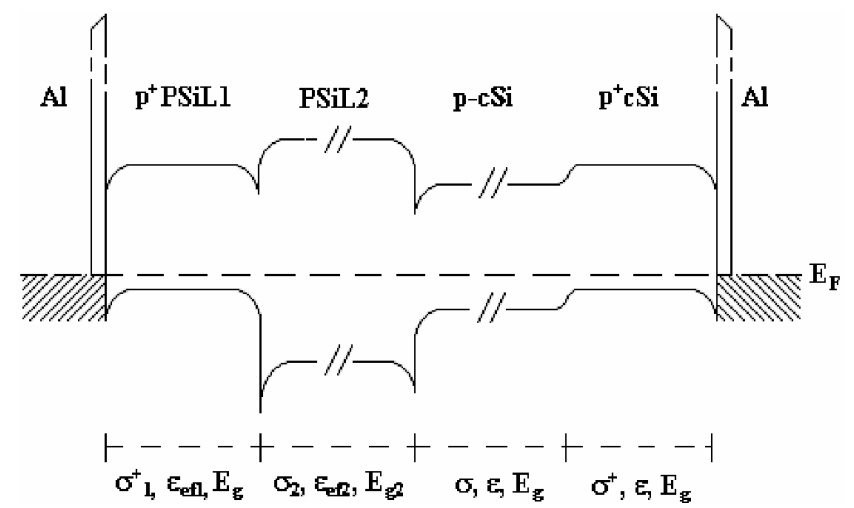

Fig. 3. Scheme of energetic bands of sample structure containing por-Si layers.

barriers exist at both sides of PSiL2 part of the structure; one as $p-p^{+}$junction of por-Si, and the another because of quantum confinement effect and widening of forbidden gap in high porosity por-Si layer $\mathrm{PSiL}_{2}$ [1]. The barriers are oriented in opposite directions, as we can see in Figure. The holes are heated by MW radiation in such a manner that smaller emf occurs firstly in the left barrier, and then, stronger emf occurs in the right barrier at high values of the MW pulse power.

In conclusion, the electric conductivity and emf of the samples containing por-Si structure have been investigated under the action of MW radiation pulses. It has been suggested that experimentally observed decrease in the resistance of the sample and rise of emf can be explained both assuming concept of carriers heating in por-Si structure by MW radiation.

\section{References}

[1] V. Dienys, J. Pozela, Hot Electrons, Mintis, Vilnius 1971, p. 289, (in Russian).

[2] V. Lehmann, Electrochemistry of Silicon, Wiley- VCH Verlag Gmb Weinheim 2002, p. 277.

[3] I.K. Itotia, R.F. Drayton, in: Proc. IEEE MTT-S Int. Microwave Symp. Digest, Ed. R. Hamilton, Co-ed N. Chiang, CD-ROM, Ed. A. Taylor, IEEE Cat. No. 02CH37278, Vol. 2, Seattle (WA0 USA), 2002, p. 681.

[4] S.P. Zhimin, Techn. Phys. Lett. 20, 55 (1994) (in Russian).

[5] S. Ašmontas, J. Gradauskas, V. Zagadsky, J. Stupakova, A. Sužiedèlis, E. Šatkovskis, Tech. Phys. Lett. 32, 603 (2006).

[6] E. Shatkovskis, J. Gradauskas, J. Stupakova, A.Česnys, A. Sužiedèlis, Lithuanian Journ. Phys. 47, 169 (2007).

[7] M. Ben-Chorin, F. Moeller, F. Koch, J. Appl. Phys. 77, 4482 (1995). 